\title{
A novel 10 glycolysis-related genes signature could predict overall survival for clear cell renal cell carcinoma
}

\author{
Qianwei Xing ${ }^{1 \dagger}$, Tengyue Zeng ${ }^{2 \dagger}$, Shouyong $\mathrm{Liu}^{2 \dagger}$, Hong Cheng ${ }^{3}$, Limin Ma ${ }^{{ }^{*}}$ and Yi Wang ${ }^{1 *}$ (D)
}

\begin{abstract}
Background: The role of glycolysis in tumorigenesis has received increasing attention and multiple glycolysisrelated genes (GRGs) have been proven to be associated with tumor metastasis. Hence, we aimed to construct a prognostic signature based on GRGs for clear cell renal cell carcinoma (ccRCC) and to explore its relationships with immune infiltration.

Methods: Clinical information and RNA-sequencing data of ccRCC were obtained from The Cancer Genome Atlas (TCGA) and ArrayExpress datasets. Key GRGs were finally selected through univariate COX, LASSO and multivariate COX regression analyses. External and internal verifications were further carried out to verify our established signature.

Results: Finally, 10 GRGs including ANKZF1, CD44, CHST6, HS6ST2, IDUA, KIF20A, NDST3, PLOD2, VCAN, FBP1 were selected out and utilized to establish a novel signature. Compared with the low-risk group, cCRCC patients in highrisk groups showed a lower overall survival (OS) rate $(P=5.548$ Ee-13) and its AUCs based on our established signature were all above 0.70 . Univariate/multivariate Cox regression analyses further proved that this signature could serve as an independent prognostic factor (all $P<0.05$ ). Moreover, prognostic nomograms were also created to find out the associations between the established signature, clinical factors and OS for CCRCC in both the TCGA and ArrayExpress cohorts. All results remained consistent after external and internal verification. Besides, nine out of 21 tumor-infiltrating immune cells (TIICS) were highly related to high- and low- risk cCRCC patients stratified by our established signature.
\end{abstract}

Conclusions: A novel signature based on 10 prognostic GRGs was successfully established and verified externally and internally for predicting OS of ccRCC, helping clinicians better and more intuitively predict patients' survival.

Keywords: Glycolysis, Overall survival, Prognosis, Signature, Clear cell renal cell carcinoma

\section{Background}

Renal cell carcinoma (RCC) incidence is second only to bladder cancer and there will be 73,750 newly estimated cases and 14,830 estimated death in the United States,

\footnotetext{
*Correspondence: wangyi_urology@163.com; ntmalimin@163.com

${ }^{+}$Qianwei Xing, Tengyue Zeng and Shouyong Liu contributed equally to this work.

'Department of Urology, Affiliated Hospital of Nantong University, No.20 West Temple Road, Nantong, Jiangsu Province 226001, China Full list of author information is available at the end of the article
}

2020 [1]. Therein, clear cell RCC (ccRCC) accounts for approximately $70-80 \%$ of the pathological subtype of RCC. Due to the insensitivity of radiotherapy or chemotherapy in RCC, its therapy is mainly surgical treatment. At present, survival rates of metastatic kidney cancer are still low. Its two-year survival is lower than $20 \%$, and about one-third of patients are found to have tumor metastasis when diagnosed $[2,3]$. Therefore, further understanding of their molecular mechanisms and

C C The Author(s). 2021 Open Access This article is licensed under a Creative Commons Attribution 4.0 International License, which permits use, sharing, adaptation, distribution and reproduction in any medium or format, as long as you give appropriate credit to the original author(s) and the source, provide a link to the Creative Commons licence, and indicate if changes were made. The images or other third party material in this article are included in the article's Creative Commons licence, unless indicated otherwise in a credit line to the material. If material is not included in the article's Creative Commons licence and your intended use is not permitted by statutory regulation or exceeds the permitted use, you will need to obtain permission directly from the copyright holder. To view a copy of this licence, visit http://creativecommons.org/licenses/by/4.0/ The Creative Commons Public Domain Dedication waiver (http://creativecommons.org/publicdomain/zero/1.0/) applies to the data made available in this article, unless otherwise stated in a credit line to the data. 
development of effective early screening and diagnosis methods are essential for improving the treatment effect and life quality for these patients.

In recent years, there have been more and more researches on the metabolic changes of tumor cells. Warburg effect is the most common and widely studied metabolic change in cancer cells, that is, in the presence of oxygen, malignant tumor cells have an inherent tendency to incompletely oxidize glucose [4]. Studies have revealed that many tumors have enhanced glucose uptake in adjacent tissues, and high glucose uptake rates are simultaneously present with suppressed glucose oxidation [4]. Glucose is converted into lactic acid during glycolysis, and cancer cells obtain maximum energy in this way. This phenomenon is ubiquitous in tumors [5], and this metabolic change is particularly noticeable in kidney cancer [6].

Accumulating evidence have reported that glycolysis-related genes (GRGs) are differently expressed in various malignant cancers and play important roles in tumorigenesis and progress. For example, studies have presented that about $90 \%$ of patients with sporadic ccRCC have mutations of VHL gene [7]. When this gene is deleted, HIF- $\alpha$ accumulates, and genes such as VEGF, PDGF, TGF- $\alpha$, and MMP are activated to participate in neovascularization formation, cell proliferation, infiltration and distant metastasis promote the development of tumors [8]. One of the key enzymes of glycolysis, hexokinase 2 (HK2), has turned out to be abnormally expressed in ccRCC and can promote cell proliferation and invasion [9]. Multiple genes, such as FBP1, PLOD2, VCAN, and CD44 have been demonstrated to participate in epithelial-mesenchymal transition (EMT) promotion of tumor metastasis [10-13]. More and more researches on the relationships between glycolysis and tumor occurrence and development have emerged. Glycolysis roles in the progress of RCC have also been confirmed. However, there is currently no prognostic model for ccRCC based on GRGs. Hence, this article is committed to establish a novel GRGs-related prognostic signature and to explore its associations with immune infiltration for predicting ccRCC patients' overall survival (OS).

\section{Methods}

\section{Data collection and identification of differentially} expressed GRGs

Clinical information and RNA-sequencing FPKM values of 539 ccRCC tumor samples and 72 normal tissues were obtained from The Cancer Genome Atlas (TCGA, https://portal.gdc.cancer.gov/) dataset, with a mixture of different histologic types of ccRCC included in this study. All raw data were preprocessed by normalization, $\log 2$ transformation and excluding average count values of genes $<1$. Differentially expressed GRGs were identified by the "Limma" package, in setting the cut-off values of $\mid \log 2$ fold change $\mid>1$ and false discovery rate (FDR) $<0.05$. In addition, the E-MTAB-1980 dataset from the ArrayExpress database (https://www.ebi.ac.uk/ arrayexpress/) including 99 ccRCC tumor samples were served as an external verification cohort.

\section{Gene ontology (GO) and Kyoto encyclopedia of genes and genome (KEGG) pathway enrichment analyses}

Through the enrichment of GO and KEGG pathway enrichment analyses, differently expressed GRGs' biological functions were comprehensively evaluated. Therein, GO analysis terms included molecular functions (MF), cellular components (CC) and biological processes (BP). All functional annotation and pathway enrichment analyses were conducted using the R package of "clusterProfiler".

\section{Protein-protein interaction (PPI) network and related module screening}

In order to evaluate the PPI network, we submitted these differentially expressed GRGs to the online tool STRING (http://www.string-db.org/) and visualized the PPI network by Cytoscape 3.7.0 software. By means of the Molecular Complex Detection (MCODE) plug-in, we screened out the top three hub modules identified from the PPI network with the cut-off criteria of a node degree $>3$, a combined score $>0.9$ and $P<0.05$.

\section{Prognostic model construction and validation}

We utilized data in the TCGA database as the training queue and the data in the ArrayExpress database as the external verification queue. Univariate Cox regression was performed to analyze associations between differently expressed GRGs and OS for ccRCC in the TCGA database, and 40 candidate genes were selected. The least absolute contraction and selection operator (LASSO) method was used to improve accuracy and to select out the optimal gene combination. Finally, 10 genes were screened out by multivariate Cox regression analysis to establish a signature. According to the genes' expression levels and their regression coefficients, a risk signature is established. The riskscore formula for each ccRCC patient was displayed as following:

$$
\text { Riskscore }=\sum_{i=1}^{n} \text { expi* } \beta i \text {. }
$$

Therein, $\beta$ represented the regression coefficient and exp. was the genes' expression levels. Taking the median riskScore in the TCGA training database as the 
threshold, the eligible ccRCC patients were classified into a high-risk and a low-risk group, and Kaplan-Meier survival curve was drawn to show the prognosis difference between the two groups of patients. Besides, our established signature was carefully evaluated in three validations sets including the external validation dataset (ArrayExpress), the internal validation dataset 1 (test 1 ) as well as the internal validation dataset 2 (test 2).

\section{Univariate/multivariate cox regression analyses and nomogram construction}

Univariate and multivariate Cox regression analyses were applied to evaluate whether our established signature and various clinical parameters could be served as an independent prognostic factor for ccRCC. Six clinical factors and riskscore were utilized to establish the prognostic nomogram, evaluating the ccRCC patients' 1-, 3- and 5-year OS. C-index and the area under the curve (AUC) were calculated to assess nomogram accuracy. Calibration charts were applied to intuitively assess nomogram prognostic ability. In addition, the prognosis nomogram was also verified in external validation cohort from ArrayExpress database.

\section{Verification of the GRGs' protein expression in HPA database and survival analysis in Kaplan-Meier plotter website}

By means of the Human Protein Atlas (HPA) dataset (http://www.proteinatlas.org/), we validated the $10 \mathrm{hub}$ GRGs' protein expression levels in ccRCC by immunohistochemical staining. Kaplan-Meier plotter website (http://kmplot.com/analysis/) was also applied to assess the prognosis of these 10 hub GRGs in ccRCC by survival analysis.

\section{Estimation of tumor-infiltrating immune cells (TIICs)}

The TIICs expression levels in all ccRCC samples were calculated by the "limma" package of $\mathrm{R}$ software. Then, algorithm with LM22 gene signature and 1000 permutations were calculated to find whether these TIICs are highly related to high- and low- groups stratified by our established signature [14]. $P$-value below 0.05 above mentioned was set as threshold.

\section{Statistical analysis}

$\mathrm{R}$ software 3.6.3 was utilized to calculate all statistical analyses. Survival curves were drawn by the KaplanMeier method with log-rank test. Univariate COX, LASSO and multivariate COX regression analyses were utilized to calculate the regression coefficient and to establish a signature. All statistical tests are bilateral. P-value below 0.05 was regarded to be statistically significant.

\section{Results \\ Identification of differently expressed GRGs}

The workflow of our study was presented in Supplement Figure S1. A total of 611 patients were extracted from the TCGA database, containing 539 renal cancer samples and 72 normal specimens with a mixture of different histologic types of ccRCC included in this study. The $\mathrm{R}$ package of "Limma" was applied to discover differentially expressed mRNAs (DE-mRNAs) with a thresholds of $\mid \log 2$ (FC) $\mid>1$ as well as FDR less than $0.05,113$ differently expressed GRGs were screened from the GRG list, including 44 down-regulated and 69 up-regulated GRGs. Their expression heatmap and volcano plot were shown in Fig. 1a-b.

\section{Functional annotation and pathway enrichment}

Results of GO and KEGG analyses presented that differently expressed GRGs were remarkably enriched in the biological processes (BP) analysis associated with carbohydrate catabolic process, pyruvate metabolic process, glycolytic process, hexose metabolic process, ATP generation from ADP, pyridine nucleotide metabolic process, glucose metabolic process, pyridine-containing compound metabolic process, nicotinamide nucleotide metabolic process pyruvate biosynthetic process. Through the molecular function (MF) analysis, they were significantly enriched in oxidoreductase activity, monosaccharide binding, coenzyme binding, carbohydrate phosphatase activity, organic acid binding, sugar-phosphatase activity carboxylic acid binding, oxidoreductase activity, NAD or NADP as acceptor, carbohydrate kinase activity, vitamin binding. Concerning the cellular component (CC), differently expressed GRGs were notably enriched in lysosomal lumen, Golgi lumen, oxidoreductase complex, vacuolar lumen, mitochondrial matrix, proteinaceous extracellular matrix, endoplasmic reticulum lumen, extracellular matrix. KEGG pathway analysis is significantly enriched in HIF-1 signaling pathway, Glycolysis/Gluconeogenesis, Carbon metabolism, Glucagon signaling pathway, Pyruvate metabolism, Fructose and mannose metabolism, Tyrosine metabolism, AMPK signaling pathway, Biosynthesis of amino acids, Galactose metabolism, Insulin signaling pathway, suggesting that these prognostic genes are indeed associated with glycolysis (Fig. 1c-d). 


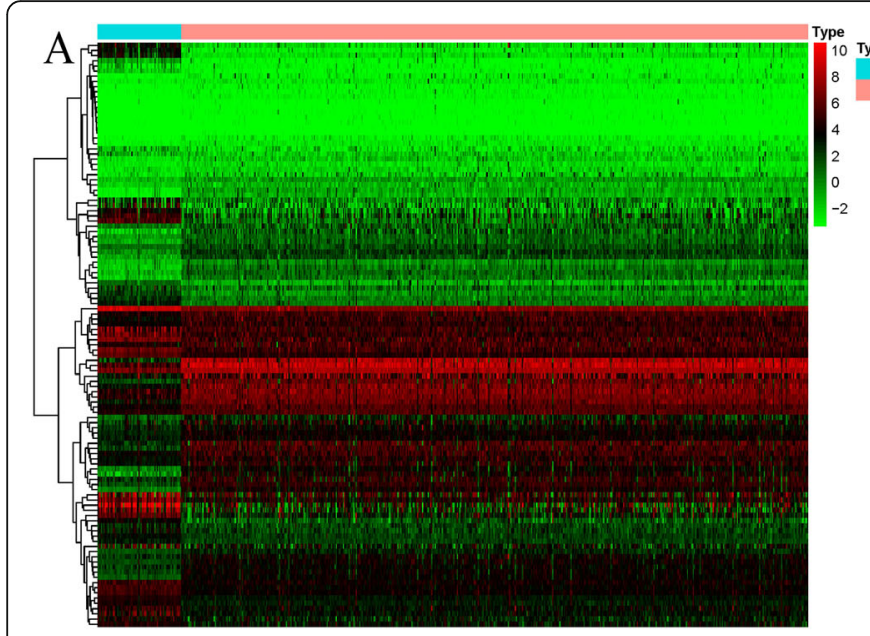

B

Volcano

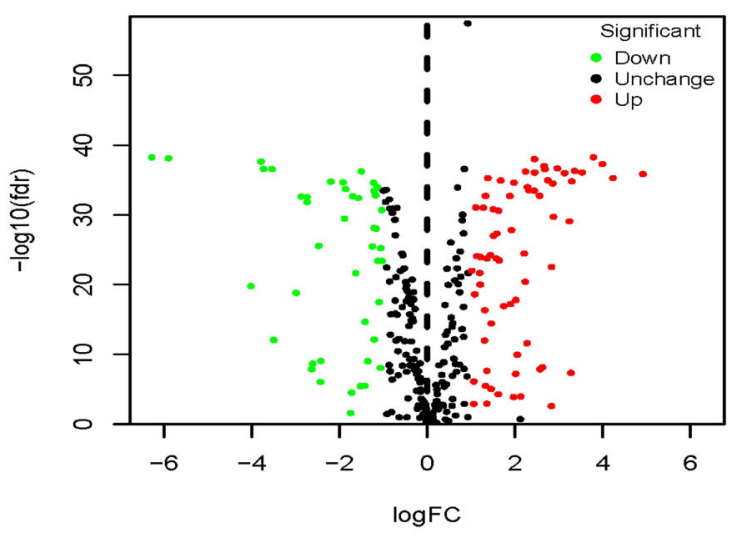

$\mathrm{C}$

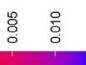

$\mathrm{D}$
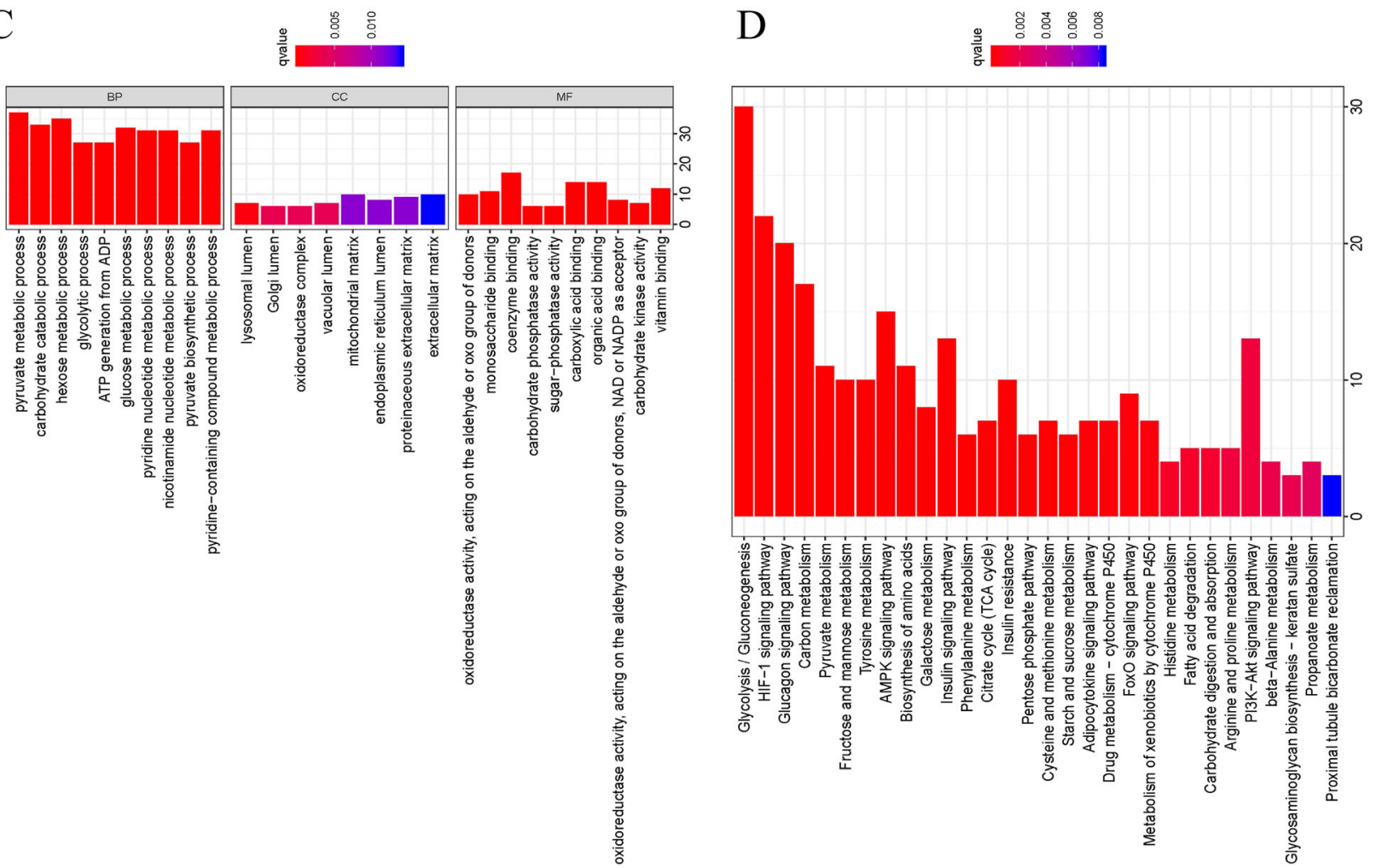

Fig. 1 The differentially expressed GRGs identified in cCRCC. a Heatmap; b Volcano plot. The red nodes represent the up-regulated genes Green nodes represent the down-regulated genes $(P$ value $<0.05$ and $|\log 2(F C)|>1$ ); $\mathbf{c} G O$ enrichment of differently expressed GRGs; $\mathbf{d}$ KEGG pathway analysis of differently expressed GRGs

PPI network integration and the top 3 key modules selection In order to further study their roles of differentially expressed GRGs in RCC, we used the STRING database to reveal the relationships between differentially expressed GRGs and visualized by Cytoscape software (Fig. 2a). We also used Cytoscape's MODE tool to process the co-expression network and to identify the top three key modules in the PPI network (Fig. 2b-d).

\section{Construction a prognostic model}

To construct a prognostic model, univariate regression analysis was done to find out 40 candidate GRGs (Fig. 3a). The LASSO Cox regression model was applied to avoid overfitting of the model (Fig. 3b-c). Finally, 10 genes were screened out by multivariate Cox regression analysis to establish a signature (Fig. 3d and Table 1). The riskscores of each patient were shown as following: 

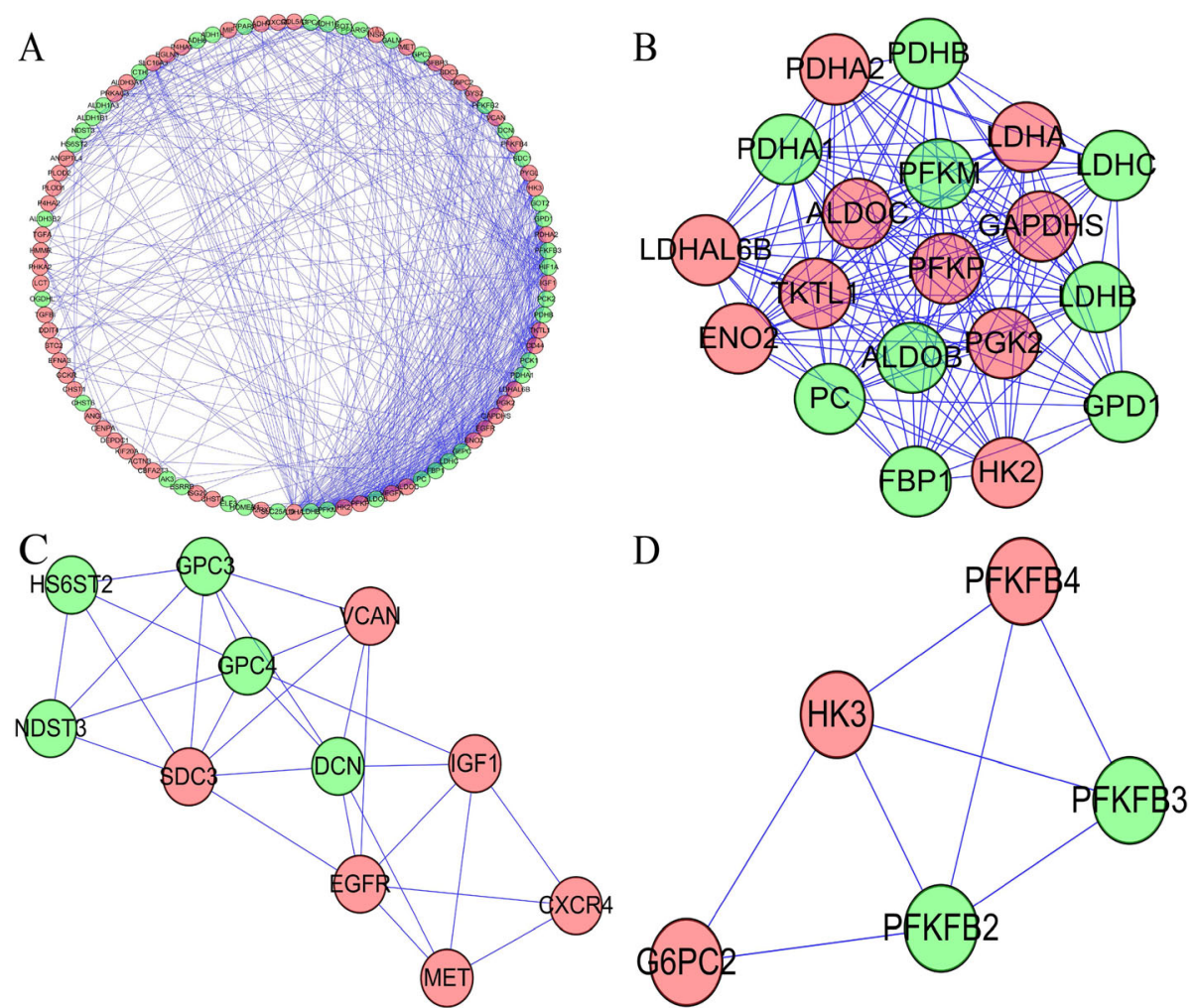

Fig. 2 Protein-protein interaction network and its top 3 key modules. a Protein-protein interaction network of differentially expressed GRGs. b-c The top three key modules from PPI network

$$
\begin{aligned}
\text { Riskscore }= & (0.0280 \times \text { Exp ANKZF1 }) \\
& +(0.0054 \times \text { Exp CD44 }) \\
& +(0.3550 \times \text { Exp CHST6 }) \\
& +(0.4116 \times \text { Exp HS6ST2 }) \\
& +(0.1155 \times \text { Exp IDUA }) \\
& +(0.1080 \times \text { Exp KIF20A }) \\
& +(-1.274 \times \text { Exp NDST3 }) \\
& +(0.0052 \times \text { Exp PLOD2 }) \\
& +(0.0059 \times \text { Exp VCAN }) \\
& +(-0.011 \times \text { Exp FBP1 }) .
\end{aligned}
$$

\section{Validation the expression and prognosis of key GRGs}

Based on our established glycolysis signature, 539 ccRCC cases were subdivided into a high-risk and a low-risk group, accordingly to the median riskscore. KaplanMeier survival analysis indicated that ccRCC patients in low-risk groups could have a markedly longer OS than patients in high-risk groups $(P=5.548 \mathrm{e}-13$, Fig. 4a). The signature showed superior predictive veracity of patients' OS with the 1-, 3- and 5-year AUC values of 0.724, 0.716 and 0.741, separately (Fig. 4b-d, Table 2). Besides, the riskscore for each sample was also calculated and ranked and the heatmap showed the expression value of ten significant GRGs between highand low-risk groups. As the risk score increased, ccRCC patients would have a shorter survival time and more dead events (Fig. 4i). Three validation datasets containing the external validation dataset, the internal validation dataset 1 as well as the internal validation dataset 2 were used to verify the risk score signature had similar predictive values in different populations. Survival analysis showed that all three testing cohorts had similar outcomes (Fig. 4e Fig. 5a and e). The 1-, 3- and 5-year AUC values of OS in three testing cohorts were all above 0.70 , also showed a favorable predictive ability (Figs. 4f-h, Fig. 5, Table 2). The expression heatmap of ten hub GRGs, survival status and risk score of signature of ccRCC patients were displayed in Fig. 5.

\section{Determination of the GRGs risk model as an independent prognostic factor}

Univariate and multivariate Cox regression analyses were applied to evaluate whether our established signature and various clinical parameters could be served as an independent prognostic factor for ccRCC. In the TCGA cohort, univariate analysis showed the glycolysis signature, age, grade, stage, $\mathrm{T}$ and $\mathrm{M}$ were all remarkably related to $O S$ (all $P<0.001$ ). Multivariate analysis 


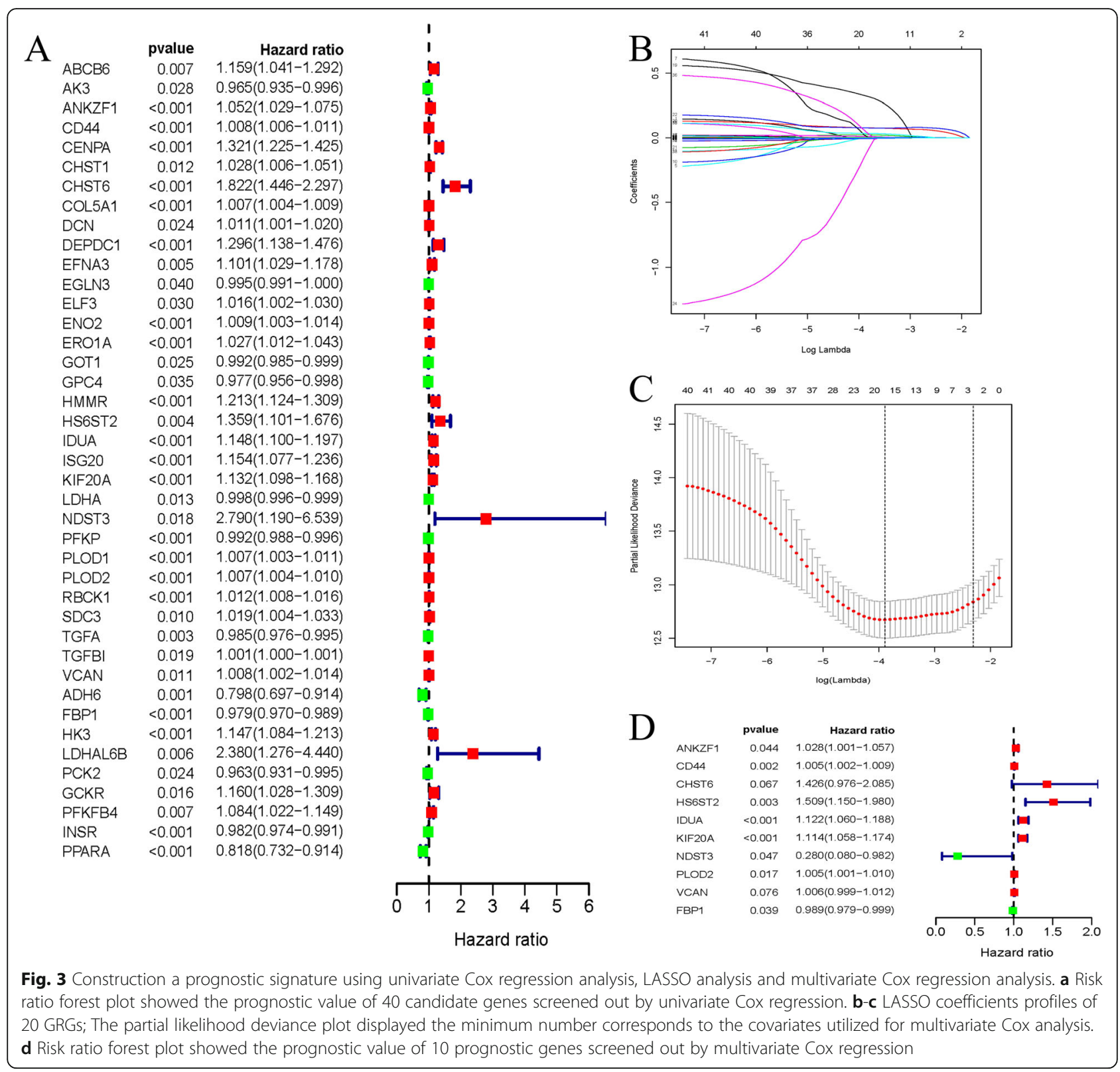

Table 1 Coefficients and HR of the 10 key prognostic GRGs

\begin{tabular}{llllll}
\hline gene & coef & HR & HR.95 L & HR.95H & p-value \\
\hline ANKZF1 & 0.02801704 & 1.02841321 & 1.0008025 & 1.05678565 & 0.0436188 \\
CD44 & 0.00539614 & 1.00541073 & 1.00198434 & 1.00884883 & 0.00194757 \\
CHST6 & 0.35495268 & 1.42611317 & 0.97559469 & 2.08467593 & 0.06689023 \\
HS6ST2 & 0.4115756 & 1.50919379 & 1.15040925 & 1.97987446 & 0.0029622 \\
IDUA & 0.11551555 & 1.12245197 & 1.06029044 & 1.18825784 & $7.07 \mathrm{E}-05$ \\
KIF2OA & 0.10802281 & 1.11407316 & 1.05755908 & 1.17360724 & $4.76 \mathrm{E}-05$ \\
NDST3 & -1.2741372 & 0.27967216 & 0.07967676 & 0.98167295 & 0.04671924 \\
PLOD2 & 0.00521398 & 1.0052276 & 1.0009296 & 1.00954405 & 0.01707989 \\
VCAN & 0.00589737 & 1.0059148 & 0.99937923 & 1.0124931 & 0.07618717 \\
FBP1 & -0.0106882 & 0.989368668 & 0.97935727 & 0.99948242 & 0.03942413 \\
\hline
\end{tabular}



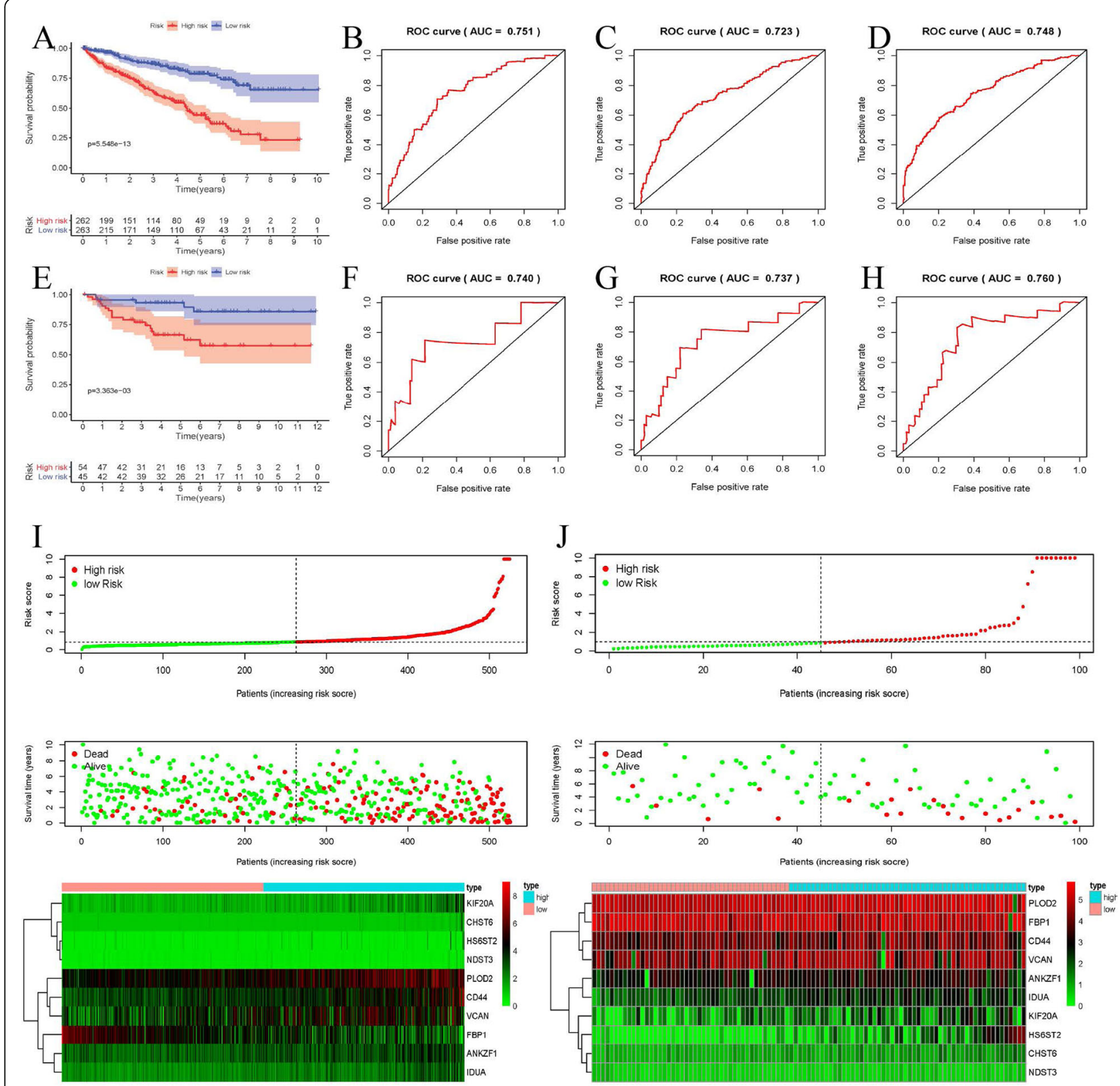

Fig. 4 Evaluation and external verification of six RBPs established signature; a Kaplan-Meier survival curves for low- and high-risk subgroups stratified by riskscore signature in the training dataset (TCGA). b-d ROC curves for forecasting 1-year, 3-year and 5-year OS based on risk score in the TCGA training dataset. e Kaplan-Meier survival curves for low- and high-risk subgroups stratified by riskscore signature in the external validation dataset (ArrayExpress); $\mathbf{f}$-h ROC curves for forecasting 1-year, 3-year and 5-year OS based on risk score in the external validation dataset (ArrayExpress); $\mathbf{i - j}$ Expression heat map, risk score distribution, and survival status in the training dataset (TCGA) and the external validation dataset (ArrayExpress)

Table 2 External and internal verification datasets of 1-year, 3-year, 5-year ROC

\begin{tabular}{llll}
\hline Dataset & 1-year ROC & 3-year ROC & 5-year ROC \\
\hline The whole training dataset (TCGA) & 0.751 & 0.723 & 0.748 \\
The external validation dataset (ArrayExpress) & 0.740 & 0.737 & 0.760 \\
The internal validation dataset 1 (test 1) & 0.739 & 0.738 & 0.752 \\
The internal validation dataset 2 (test 2) & 0.737 & 0.700 & 0.746 \\
\hline
\end{tabular}



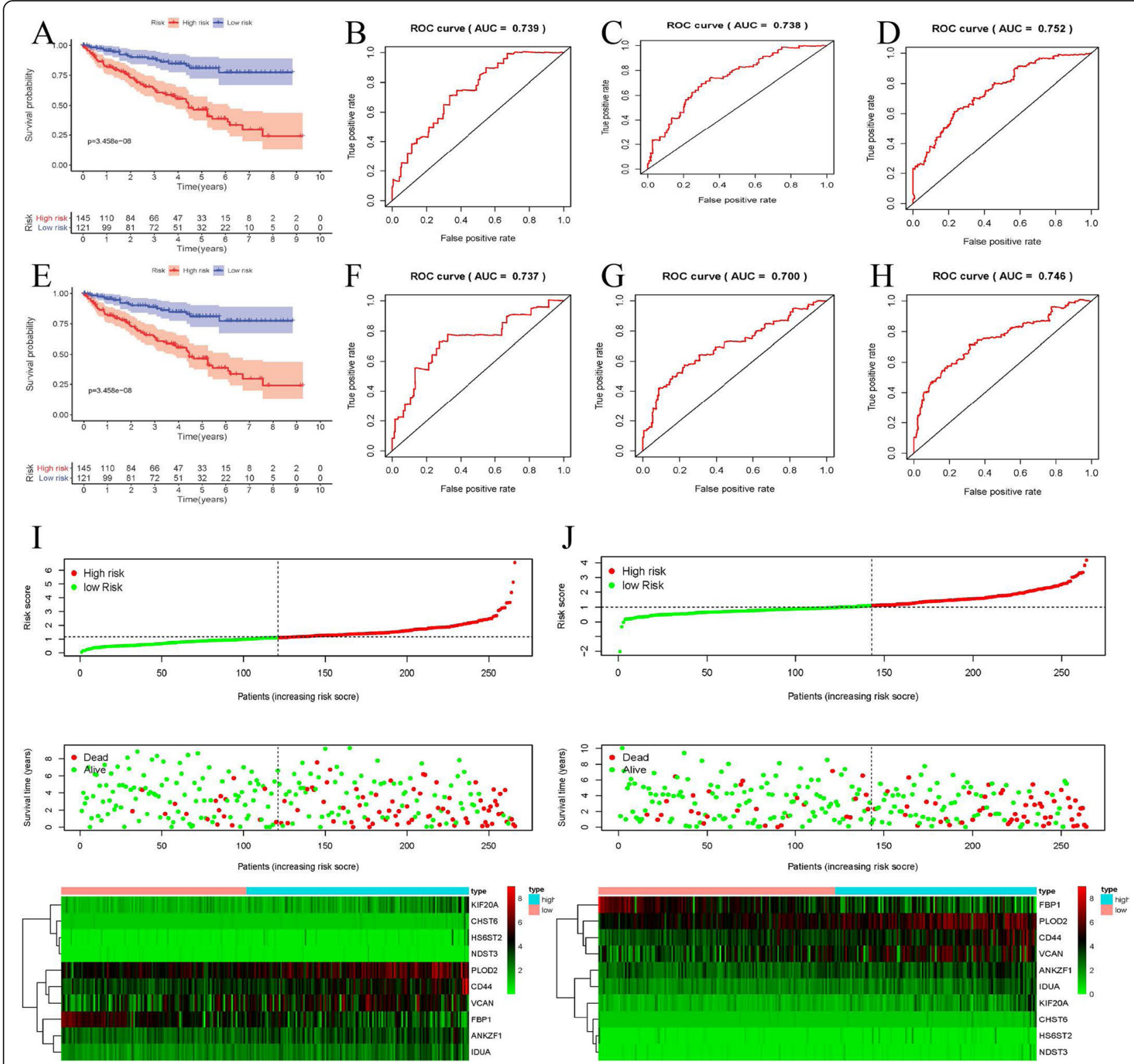

Fig. 5 Internal verification of 10 GRGs based riskscore signature. a Kaplan-Meier survival curves for low- and high-risk subgroups stratified by riskscore signature in the internal validation dataset $1 ; \mathbf{b}$-d ROC curves for forecasting 1-year, 3-year and 5-year OS based on risk score in the internal validation dataset 1; e Kaplan-Meier survival curves for low- and high-risk subgroups stratified by riskscore signature in the internal validation dataset 2; $\mathbf{f - h}$ ROC curves for forecasting 1-year, 3-year and 5-year OS based on risk score in the internal validation dataset 2; i-j Expression heat map, risk score distribution, and survival status in the internal validation dataset 1 and in the internal validation dataset 2

indicated that the glycolysis signature, age, grade and stage were all marked correlated with OS (all $P<0.01$ ). Therefore, the prognostic glycolysis signature constructed by the TCGA training set was an independent prognostic factor for ccRCC (Fig. 6, Table 3).

\section{Nomogram construction based on the clinical characteristic and ten GRGs' signature}

To predict ccRCC patients' prognosis, a prognostic nomogram was constructed by TCGA dataset to predict the 1-, 3- and 5-year OS for ccRCC. Severn prognostic parameters were included in the prediction model, including the riskScore, age, gender, grade, $\mathrm{T}, \mathrm{M}$ and $\mathrm{N}$ (Fig. 7a). The nomogram showed good predictive power of 1-, 3- and 5-year OS rates for ccRCC. The 1-, 3- and 5-year AUC and their C-index were 0.836, 0.799, 0.765 and 0.781 in the TCGAs database respectively (Table 4 , Supplement Figure S2). Calibration charts (Fig. 7c) showed that the 1-, 3- and 5-year survival rates of the TCGA cohort predicted were in excellently consistent 


\begin{tabular}{|lrr}
\hline $\begin{array}{l}\text { A } \\
\text { age }\end{array}$ & pvalue & Hazard ratio \\
gender & 0.001 & $1.031(1.017-1.045)$ \\
race & 0.534 & $1.176(0.706-1.959)$ \\
grade & $<0.001$ & $1.972(1.641-2.369)$ \\
stage & $<0.001$ & $1.880(1.665-2.124)$ \\
T & $<0.001$ & $2.043(1.724-2.421)$ \\
M & $<0.001$ & $2.136(1.689-2.701)$ \\
N & 0.063 & $0.862(0.737-1.008)$ \\
riskScore & $<0.001$ & $1.032(1.022-1.042)$
\end{tabular}

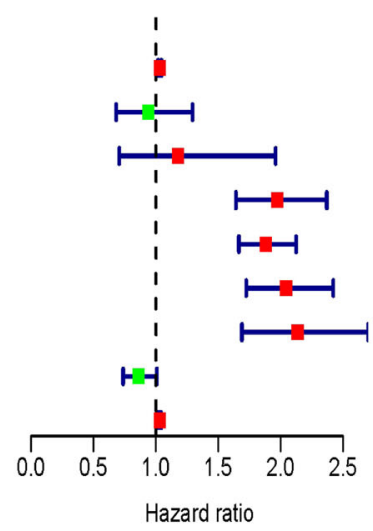

B

$\begin{array}{lrr} & \text { pvalue } & \text { Hazard ratio } \\ \text { age } & <0.001 & 1.033(1.017-1.048) \\ \text { gender } & 0.667 & 0.930(0.669-1.293) \\ \text { race } & 0.676 & 1.127(0.642-1.978) \\ \text { grade } & 0.002 & 1.404(1.127-1.749) \\ \text { stage } & 0.005 & 1.615(1.154-2.261) \\ \text { T } & 0.316 & 1.151(0.874-1.515) \\ \text { M } & 0.969 & 0.988(0.536-1.819) \\ \text { N } & 0.051 & 0.851(0.724-1.001) \\ \text { riskScore } & <0.001 & 1.021(1.011-1.032)\end{array}$

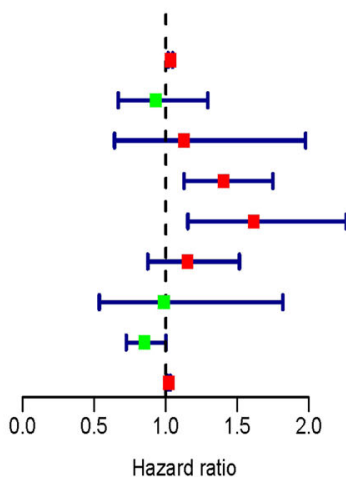

Fig. 6 Independent prognostic factor evaluation. a Univariate cox regression analysis of the training dataset (TCGA). b Multivariate cox regression analysis of the training dataset (TCGA)

with actual observations. In addition, we constructed another prognosis nomogram as an external verification set to verify the previous results in ArrayExpress dataset (Fig. 7b). Its C-index was 0.86 and the AUC values were 0.888, 0.915, and 0.902 (Table 4, Supplement Figure S2) respectively, indicating that the nomogram also has good predictive power for OS rates in the testing set. The calibration chart shows excellent agreement between the 1-, 3- and 5-year OS predictions and actual observations of ccRCC patients in the external testing set (Fig. 7d).

\section{Prognostic value of 10 key GRGs and their associations} between our established signature and clinical factors Based on the median expression, survival analyses of 10 GRGs (ANKZF1, CD44, CHST6, HS6ST2, IDUA, KIF20A, NDST3, PLOD2, VCAN, FBP1) by KaplanMeier Plotter website were displayed in Fig. 8. The correlations between our established and clinical factors were also analyzed and revealed that the established signature was firmly associated with grade $(P=0.030)$, stage
$(P=0.036)$ and staged T $(P=0.042)$ (Table 5, Supplementary Figure S3).

\section{Validation of the expression of 10 critical GRGs in ccRCC from HPA database}

As detailed in Supplementary Figure S4, immunohistochemical staining of these 10 critical GRGs from HPA database were utilized to verify their protein expressions. Compared with normal kidney tissues, antibody stainings for ANKZF1, CD44, IDUA, KIF20A, PLOD2, and VCAN were high in ccRCC tumor tissues, whereas they were low for CHST6, HS6ST2, NDST3 and FBP1.

\section{Clinical factors stratified by the riskScore for OS}

Our results indicated that our established riskScore was capable of predicting OS in age $\leq 65$, age $>65$, Female, Male, Grade 1-2, Grade 3-4, M0, N0, White, Stage I-II, Stage III-IV, T1-2 stage, T3-4 stage (all $P<0.01$; Fig. 9).

Table 3 Univariate and multivariate Cox regression analysis of external and internal verification datasets for overall survival (OS) in TCGA training dataset

\begin{tabular}{|c|c|c|c|c|c|c|c|c|}
\hline \multirow[t]{2}{*}{ id } & \multicolumn{4}{|c|}{ Univariate Cox regression analysis } & \multicolumn{4}{|c|}{ Multivariate Cox regression analysis } \\
\hline & HR & HR.95 L & HR.95H & pvalue & HR & HR.95 L & HR.95H & $p$ value \\
\hline age & 1.030913 & 1.017301 & 1.044707 & 7.14E-06 & 1.032813 & 1.01749 & 1.048366 & $2.30 \mathrm{E}-05$ \\
\hline gender & 0.939895 & 0.682968 & 1.293475 & 0.703594 & 0.9303 & 0.669431 & 1.292828 & 0.666978 \\
\hline race & 1.175718 & 0.705799 & 1.958508 & 0.534112 & 1.127239 & 0.642321 & 1.978247 & 0.676404 \\
\hline grade & 1.971561 & 1.640971 & 2.368751 & $4.20 \mathrm{E}-13$ & 1.403862 & 1.126968 & 1.74879 & 0.002475 \\
\hline stage & 1.880446 & 1.664529 & 2.12437 & $3.38 \mathrm{E}-24$ & 1.615246 & 1.154106 & 2.260642 & 0.00518 \\
\hline $\mathrm{T}$ & 2.043146 & 1.72421 & 2.421079 & $1.57 \mathrm{E}-16$ & 1.150896 & 0.874324 & 1.514955 & 0.316239 \\
\hline M & 2.135679 & 1.688608 & 2.701115 & $2.42 \mathrm{E}-10$ & 0.987786 & 0.536484 & 1.81873 & 0.968524 \\
\hline N & 0.862088 & 0.737405 & 1.007852 & 0.062627 & 0.85109 & 0.723966 & 1.000537 & 0.050765 \\
\hline riskScore & 1.031719 & 1.021717 & 1.041819 & 3.33E-10 & 1.021333 & 1.010815 & 1.03196 & $6.42 \mathrm{E}-05$ \\
\hline
\end{tabular}




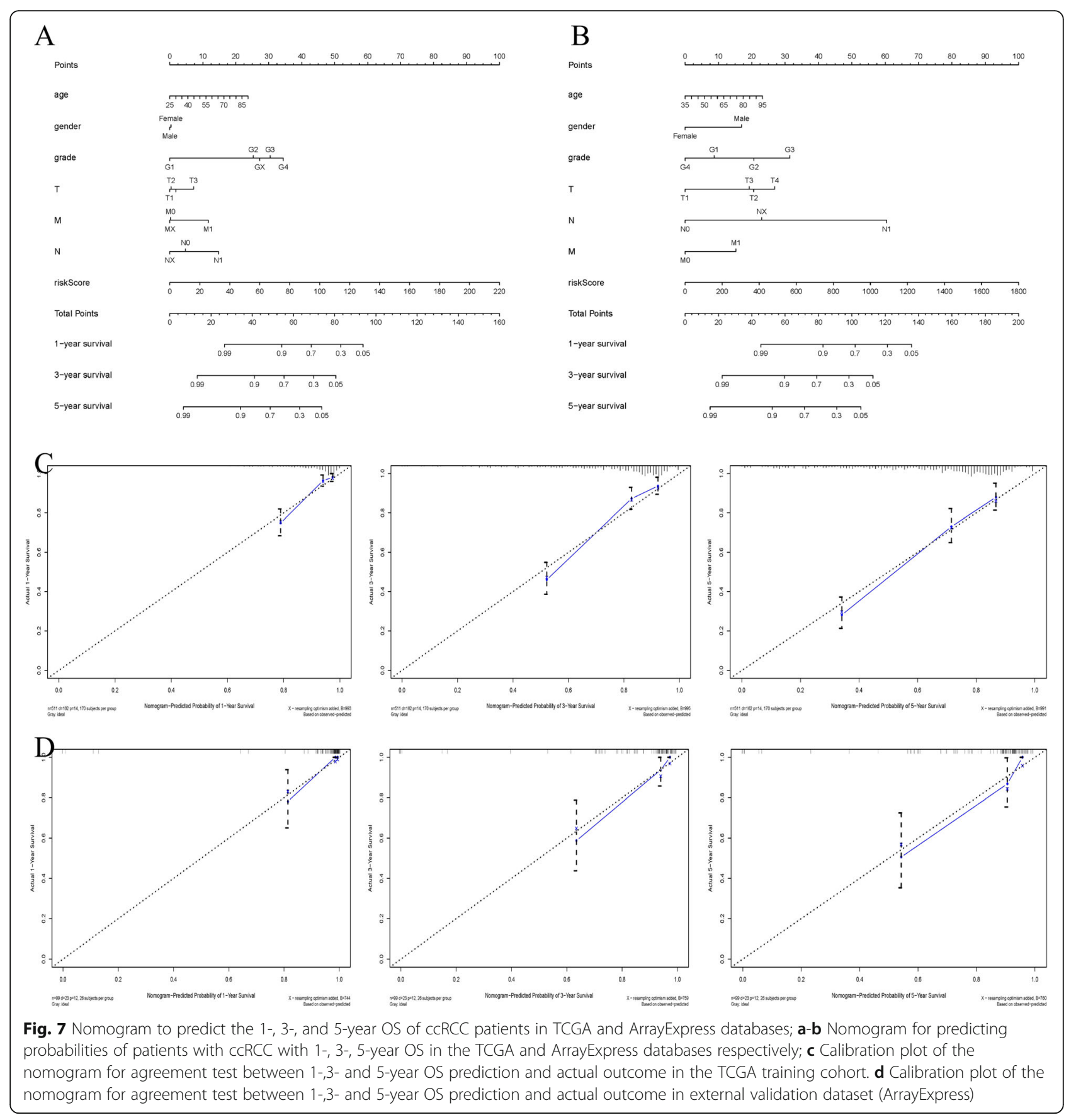

Table 4 1-year, 3-year, 5-year ROC and C-index of nomogram for in the training dataset (TCGA) and the external validation dataset (ArrayExpress)

\begin{tabular}{lllll}
\hline & 1-year ROC & 3-year ROC & 5-year ROC & C-index \\
\hline TCGA cohort & 0.836 & 0.799 & 0.765 & 0.781 \\
ArrayExpress cohort & 0.888 & 0.915 & 0.902 & 0.868 \\
\hline
\end{tabular}

\section{Associations between TIICs and our established signature for ccRCC}

As displayed in Fig. 10a-i, nine out of 21 TIICs (T cells CD4 memory activated, Plasma cells, $\mathrm{T}$ cells gamma delta, $\mathrm{T}$ cells regulatory (Tregs), Macrophages M0, Monocytes, Macrophages M1, Mast cells resting, Dendritic cells resting) were highly associated with high- and low- risk ccRCC patients stratified by our established riskScore (all $P<0.05$ ). As detailed in Fig. 10j, radar chart showed the relationships of 21 TIICs between 

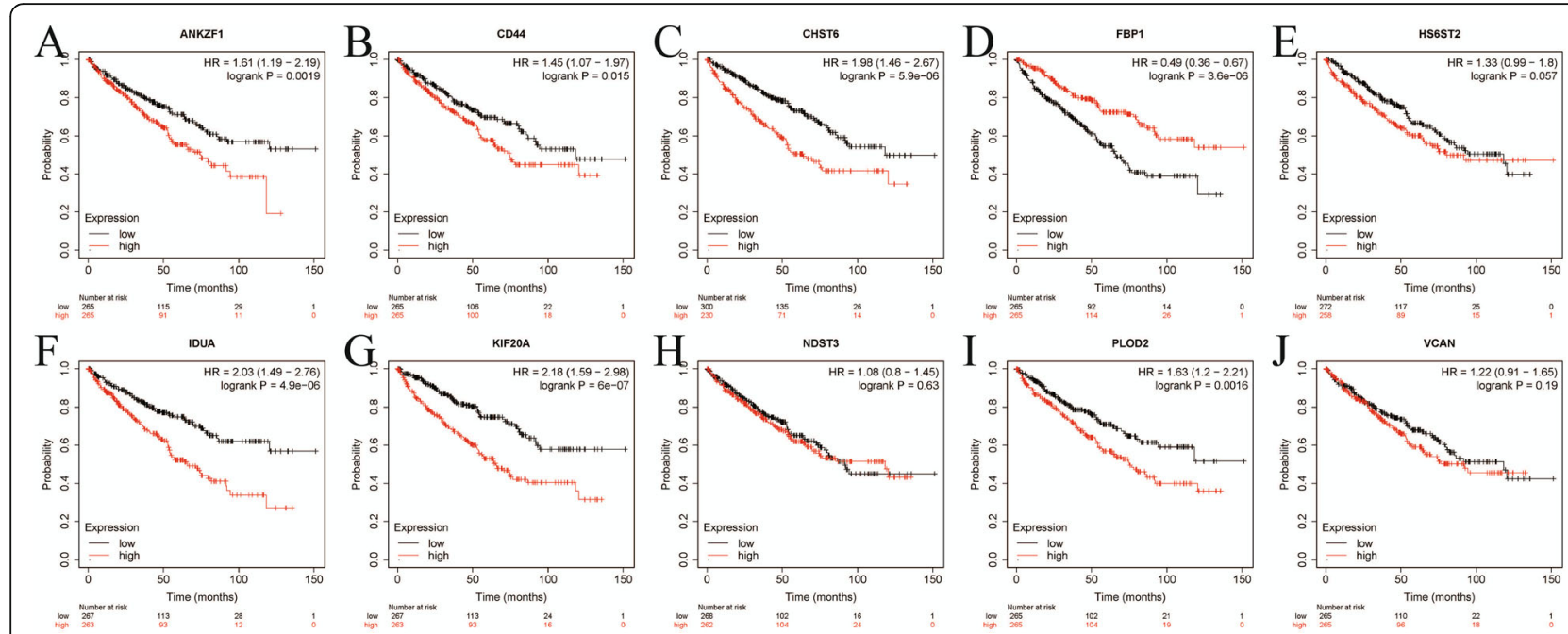

Fig. 8 Survival analyses of 10 GRGs by Kaplan-Meier Plotter website; a ANKZF1; b CD44; c CHST6; d FBP1; e HS6ST2; f IDUA; $g$ KIF2OA; h NDST3; i PLOD2; $\mathbf{j}$ VCAN

high-risk and low-risk ccRCC patients and nine out of these 21 TIICs were statistically significant (all $\mathrm{P}<0.05$ ).

\section{Discussion}

According to GLOBOCAN statistics, there were approximately 403,262 new cases of kidney cancers and 175,098 death worldwide in 2018 [15]. Of all human malignancies, kidney cancer accounted for about 2-3\% [2]. At present, few studies had focused on the expression pattern of GRGs and their roles in predicting ccRCC survival. Hence, we identified differentially expressed GRGs between tumor samples and normal tissues based on TCGA raw data to construct a PPI network and to perform GO and KEGG pathway enrichment analysis. In addition, we also conducted univariate COX, LASSO and multivariate COX regression analyses to establish a signature for predicting the ccRCC patients' prognosis based on 10 prognostic-related GRGs and further

Table 5 Clinical correlation analysis between 10 prognostic GRGs, riskscore and clinical features

\begin{tabular}{|c|c|c|c|c|c|c|c|c|}
\hline id & age & gender & race & grade & stage & $\mathrm{T}$ & M & $\mathrm{N}$ \\
\hline ANKZF1 & $\begin{array}{l}-0.511 \\
(0.610)\end{array}$ & $0.375(0.708)$ & $5.65(0.059)$ & $-2.764(0.006)$ & $-3.114(0.002)$ & $-2.766(0.006)$ & $-3.05(0.003)$ & $\begin{array}{l}-0.075 \\
(0.940)\end{array}$ \\
\hline CD44 & $\begin{array}{l}-0.342 \\
(0.733)\end{array}$ & $\begin{array}{l}-2.495 \\
(0.013)\end{array}$ & $4.916(0.086)$ & $-2.216(0.027)$ & $-2.172(0.031)$ & $-2.268(0.024)$ & $-1.832(0.070)$ & $0.795(0.427)$ \\
\hline CHST6 & $\begin{array}{l}-1.198 \\
(0.233)\end{array}$ & $-0.024(0.981)$ & $5.346(0.069)$ & $-1.743(0.082)$ & $-1.794(0.074)$ & $-1.872(0.063)$ & $0.193(0.847)$ & $0.581(0.561)$ \\
\hline HS6ST2 & $\begin{array}{l}-0.236 \\
(0.814)\end{array}$ & $1.4(0.163)$ & $1.036(0.596)$ & $0.206(0.837)$ & $-0.313(0.755)$ & $-0.593(0.554)$ & $-0.12(0.905)$ & $\begin{array}{l}-0.394 \\
(0.694)\end{array}$ \\
\hline IDUA & $\begin{array}{l}-0.556 \\
(0.579)\end{array}$ & $1.105(0.270)$ & $13.523(0.001)$ & $\begin{array}{l}-4.243(2.673 e- \\
05)\end{array}$ & $\begin{array}{l}-4.079(5.649 e- \\
05)\end{array}$ & $\begin{array}{l}-3.349(9.142 \mathrm{e}- \\
04)\end{array}$ & $\begin{array}{l}-3.78(2.389 e- \\
04)\end{array}$ & $\begin{array}{l}-0.132 \\
(0.895)\end{array}$ \\
\hline KIF20A & $\begin{array}{l}-0.84 \\
(0.402)\end{array}$ & $\begin{array}{l}-2.636 \\
(0.009)\end{array}$ & $\begin{array}{l}16.433(2.701 \mathrm{e}- \\
04)\end{array}$ & $\begin{array}{l}-5.354(1.566 \mathrm{e}- \\
07)\end{array}$ & $\begin{array}{l}-4.874(1.993 e- \\
06)\end{array}$ & $\begin{array}{l}-4.542(9.366 \mathrm{e}- \\
06)\end{array}$ & $-2.609(0.010)$ & $\begin{array}{l}-0.112 \\
(0.911)\end{array}$ \\
\hline NDST3 & $\begin{array}{l}-0.454 \\
(0.650)\end{array}$ & $-0.447(0.655)$ & $0.183(0.912)$ & $-2.118(0.035)$ & $-1.423(0.156)$ & $-1.434(0.153)$ & $-1.32(0.189)$ & $\begin{array}{l}2.511 \\
(0.013)\end{array}$ \\
\hline PLOD2 & $\begin{array}{l}0.171 \\
(0.865)\end{array}$ & $\begin{array}{l}-2.622 \\
(0.009)\end{array}$ & $9.262(0.010)$ & $-2.86(0.004)$ & $\begin{array}{l}-4.439(1.319 \mathrm{e}- \\
05)\end{array}$ & $\begin{array}{l}-4.286(2.663 e- \\
05)\end{array}$ & $-2.177(0.031)$ & $1.353(0.177)$ \\
\hline VCAN & $\begin{array}{l}-0.054 \\
(0.957)\end{array}$ & $\begin{array}{l}-2.013 \\
(0.045)\end{array}$ & $5.449(0.066)$ & $-1.422(0.156)$ & $-1.507(0.133)$ & $-1.453(0.147)$ & $-0.316(0.752)$ & $0.947(0.344)$ \\
\hline FBP1 & $\begin{array}{l}1.325 \\
(0.186)\end{array}$ & $\begin{array}{l}4.761(3.75 e- \\
06)\end{array}$ & $5.32(0.070)$ & $3.295(0.001)$ & $\begin{array}{l}3.412(7.026 \mathrm{e}- \\
04)\end{array}$ & $3.303(0.001)$ & $0.967(0.335)$ & $0.976(0.330)$ \\
\hline riskScore & $\begin{array}{l}-1.338 \\
(0.183)\end{array}$ & $-1.399(0.163)$ & $0.705(0.703)$ & $-2.178(0.030)$ & $-2.111(0.036)$ & $-2.048(0.042)$ & $-0.851(0.396)$ & $\begin{array}{l}-0.655 \\
(0.513)\end{array}$ \\
\hline
\end{tabular}




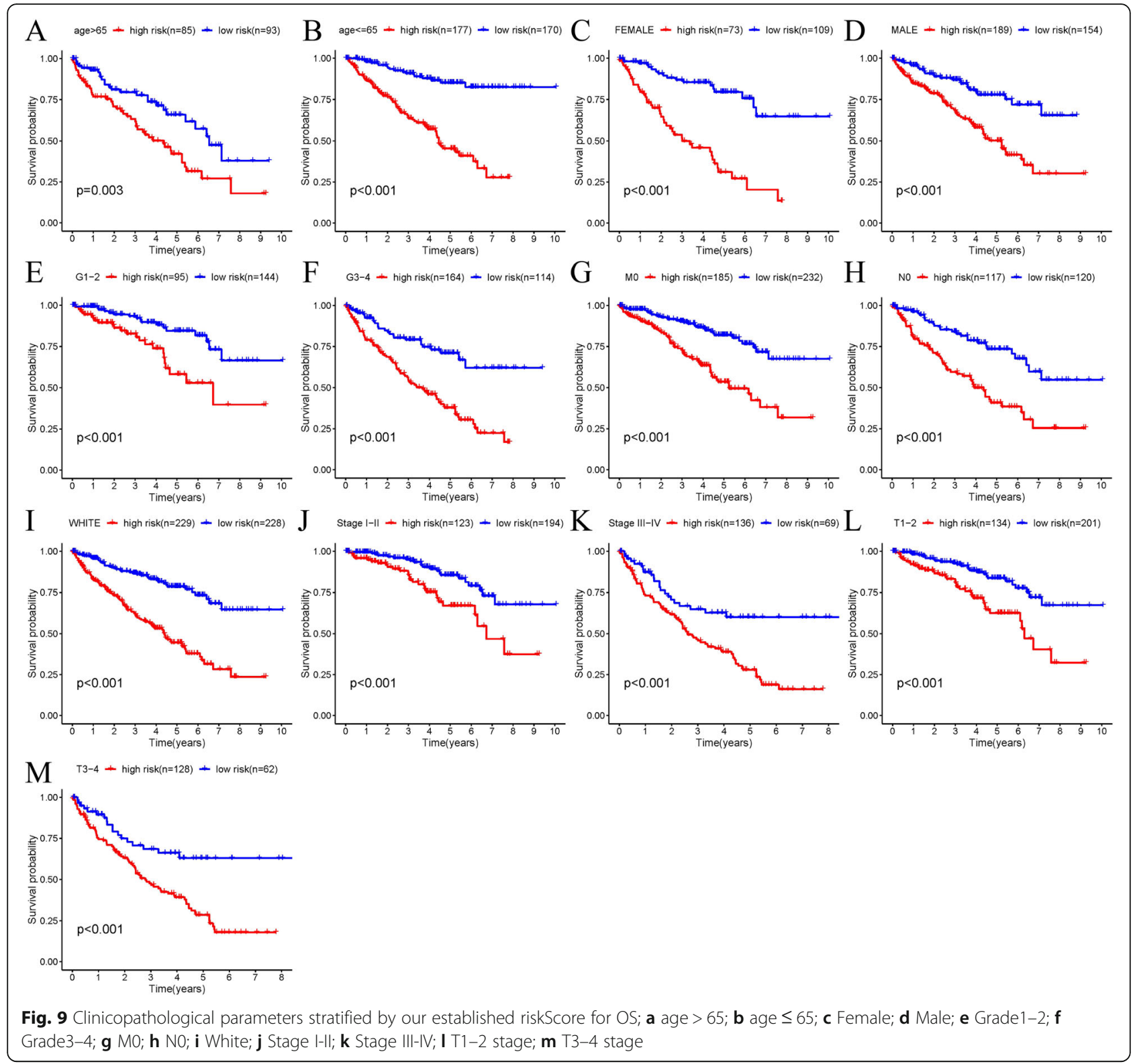

explored its associations with immune infiltration. These works may help to identify novel effective biomarkers for ccRCC prognosis.

According to reports, there are metabolic changes in a variety of cancers, and glycolysis is the most common and widely studied metabolic change [16]. It was the first time for us to identify differently expressed GRGs and then screen out 10 prognostic-related GRGs (ANKZF1, CD44, CHST6, HS6ST2, IDUA, KIF20A, NDST3, PLOD2, VCAN, FBP1), based on TCGA, by univariate/ multivariate Cox regression and LASSO regression analyses. Some studies have shown that these GRGs play important roles in the tumorigenesis and progress of various tumors, including renal clear cell carcinoma. For example, the expression of HS6ST2 was up-regulated in tumors of gastric cancer, and related to the poor prognosis [17]. Liep J et al. found that overexpression of miR-145-5p and miR-141-3p could inhibit the migration and invasion of RCC cells by influencing the HS6ST2 expression [18]. Regarding KIF20A, it was reported to be overexpressed in various tumors such as bladder cancer, cervical cancer, glioma, lung adenocarcinoma, ovarian clear cell carcinoma, colorectal cancer, liver cancer, prostate cancer, gastric cancer, etc., and often indicates a poor prognosis and poor clinicopathological features [19-28]. Studies had shown that KIF20A might promote the proliferation, invasion and migration of tumor cells by activating the JAK2/STAT3 pathway $[23,26]$. Asahara $S$ et al. has also developed cancer vaccine reagent KIF20-66 for the treatment of pancreatic cancer, which 

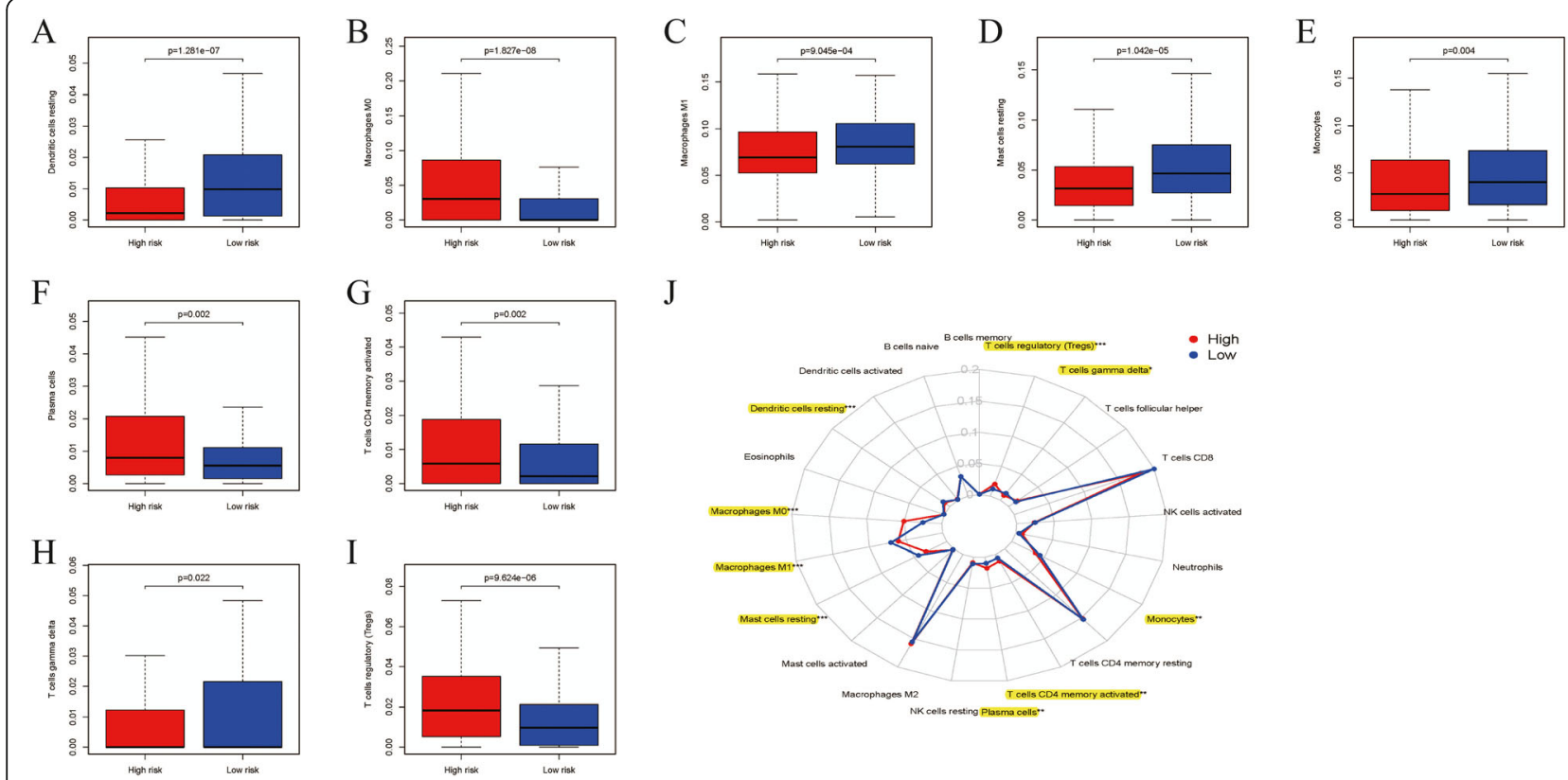

J

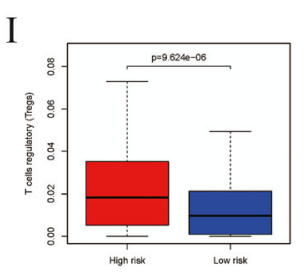

Fig. 10 Associations between tumor-infiltrating immune cells (TIICs) and GRGs based riskscore signature in ccRCC; a Dendritic cells resting between high- and low-risk ccRCC patients; b Macrophages M0 between high- and low-risk ccRCC patients; c Macrophages M1 between highand low-risk ccRCC patients; d Mast cells resting between high- and low-risk ccRCC patients; e Monocytes between high- and low-risk ccRCC patients; $\mathbf{f}$ Plasma cells between high- and low-risk ccRCC patients; $\mathbf{g}$ T cells CD4 memory activated between high- and low-risk ccRCC patients; $\mathbf{h}$ T cells gamma delta between high- and low-risk ccRCC patients; i T cells regulatory (Tregs) between high- and low-risk ccRCC patients; $\mathbf{j}$ Radar chart showed the difference of immune cell infiltration abundances in ccRCC subtypes

has a beneficial therapeutic effect on advanced pancreatic cancer [29]. Epithelial mesenchymal transformation (EMT) is one of the key steps that cause distant metastasis of tumors [30]. FBP1, PLOD2, VCAN, CD44 are all proved to relate to EMT. Studies have shown that PLOD2 is regulated by many factors, such as HIF- $1 \alpha$, TGF- $\beta$ and microRNA [31]. FBP1 is a rate-limiting enzyme for gluconeogenesis and has recently been considered as a tumor suppressor for various cancers [32]. It is proved that FBP1 interacts with HIF to inhibit the function of nuclear HIF, inhibit glycolysis and pentose phosphate pathway and inhibit the proliferation of renal cancer cells [10]. FBP1 overexpression inhibits the proliferation, migration, invasion and tumorigenesis of cholangiocarcinoma cells by inhibiting the $\mathrm{Wnt} / \beta$ pathway [33]. FBP1 gene silencing could activate the MAPK pathway and then promote cell EMT, invasion and metastasis in prostate cancer [34]. Several studies have found that PLOD2 induces epithelial-mesenchymal transition (EMT) mainly through the PI3K / AKT signaling pathway [11]. Mitsui Y et al. found that VCAN promotes tumorigenesis and metastasis of ccRCC [35]. VCAN knockdown significantly reduced the proliferation of renal cancer cells and increased apoptosis, which is linked to the changes of several TNF signaling related genes such as TNF $\alpha$, BID and BAK [35]. TGF- $\beta$ had the ability to enhance the invasiveness of ovarian cancer cells by up-regulating VCAN in fibroblasts (CAF) [36]. Up-regulated VCAN could also promote the invasion and movement of ovarian cancer cells by up-regulating $\mathrm{CD} 44$ and activating the NF- $\mathrm{kB}$ signaling pathway, matrix metalloproteinase 9 and hyaluronan-mediated movement receptors [36]. Wu et al. have shown that CD44 + bladder cancer cells have a higher invasive ability [13].

Current research shows that clinical and pathological features such as age and metastasis are not sufficient to accurately evaluate cancer patients' survival. At present, we are looking for effective biomarkers to predict the survival of cancer patients. However, single gene expression levels will be affected by many factors, making these markers unreliable to be independent prognostic indicators. Therefore, a statistical model composed of multiple related genes is much more accurate in evaluating the prognosis of tumor patients than using a single biomarker, so this model has been extensively used. Few studies have concentrated on the role of glycolysis in the prediction of ccRCC prognosis. We established a new prognostic signature based on the expression of 10 GRGs. Based on this risk scoring model, ccRCC patients were classified into a high-group and low-risk group. Patients with high-risk scores had significantly lower OS compared to those with low-risk scores. In addition, we combined the established riskscore and multiple clinical 
parameters to construct a nomogram to predict 1-, 3-, and 5-year OS in ccRCC patients. The calibration chart based on the TCGA database shows that the predicted value and the observed value are very close, indicating that the prediction performance of nomogram is very good. Similarly, it is checked in the external verification set and the two internal verification sets. Therefore, our new prognosis nomogram may be better than the original clinical factors to help clinicians predict the survival status for ccRCC and provide specific individualized treatment.

As far as we knew, this was the first signature to predict the OS of ccRCC patients based on GRGs. We successfully established a risk signature based on GRGs and verified it in three verification sets including one external verification data set (ArrayExpress) and two internal verification data sets (test 1 and test 2 ). Our results remained stable both internally and externally. Of course, this study also had several limitations. Firstly, as a bioinformatics analysis article, our research is retrospective and the clinical information downloaded from the online database was incomplete and limited. Secondly, 10 glycolysis-related genes had not been experimentally verified and our constructed nomogram had not been validated by our own data. Thirdly, due to limited clinical information, we had difficulties in comparing our established signature with other available tools in the clinic, such as the Heng score (IMDC).

\section{Conclusions}

Taken together, 10 GRGs including ANKZF1, CD44, CHST6, HS6ST2, IDUA, KIF20A, NDST3, PLOD2, VCAN, FBP1 were selected out and utilized to establish a novel signature. The GRGs based signature was successfully established and verified externally and internally for predicting OS, helping clinicians better and more intuitively predict ccRCC patients' survival. As an independent prognostic factor, our established signature showed excellent predictive efficacy for ccRCC and significantly associated with immune infiltration. Further researches were required to verify our findings.

\footnotetext{
Abbreviations

GRGs: Glycolysis-related genes; ccRCC: Clear cell renal cell carcinoma; TCGA: The Cancer Genome Atlas; OS: Overall survival; FDR: False discovery rate; FC: Fold change; GO: Gene ontology; KEGG: Kyoto encyclopedia of genes and genome; BP: Biological processes; MF: Molecular functions; CC: Cellular components; PPI: Protein-protein interaction; LASSO: Least absolute contraction and selection operator; HPA: Human Protein Atlas; TIICs: Tumor-infiltrating immune cells
}

\section{Supplementary Information}

The online version contains supplementary material available at https://doi. org/10.1186/s12885-021-08111-0
Additional file 1: Supplement Figure S1. Workflow chart for identifying the glycolysis signature associated with CCRCC survival.

Additional file 2: Supplement Figure S2. Time-dependent ROC analyses for OS prediction by nomogram including1-, 3-, 5-year in both TCGA and ArrayExpress databases. (A-C) 1-, 3-, 5-year in TCGA dataset; (D-F) 1-, 3-, 5-year in ArrayExpress dataset.

Additional file 3: Supplement Figure S3. Association between clinicopathologic characteristics (grade, stage, T) and our established riskScore; (A) Distribution of riskscores in grade; (B) Distribution of riskscores in stage; (C) Distribution of riskscores in $\mathrm{T}$ stage.

Additional file 4: Supplementary Figure S4. Validation of the expression of 10 critical GRGs in cCRCC from HPA database; (A) ANKZF1; (B) CD44; (C) CHST6; (D) FBP1; (E) HS6ST2; (F) IDUA; (G) KIF20A; (H) NDST3; (I) PLOD2; (J) VCAN.

\section{Acknowledgements}

We would like to thank the researchers and study participants for their contributions.

\section{Authors' contributions}

Y.W and LM.M conceived and designed the study; H.C acquired and analyzed the data; QW.X and TY.Z interpreted the data and drafted the manuscript; SY.L analyzed the data and modified the manuscript. All authors have read and approved the final published manuscript.

\section{Funding}

This article was funded by the National Natural Science Foundation of China [grant number: 81771571] and the Postdoctoral Science Foundation of Jiangsu Province: 2020Z071. The funder had no role in study design, analysis, interpretation of data, the writing of the manuscript and the decision to submit the article for publication.

\section{Availability of data and materials}

The RNA-sequencing data of CCRCC samples and normal kidney tissue samples with corresponding clinical information were downloaded from the Cancer Genome Atlas (TCGA) database (https://portal.gdc.cancer.gov/) and the ArrayExpress database (E-MTAB-1980, https://www.ebi.ac.uk/arrayexpress/ ).

\section{Declarations}

Ethics approval and consent to participate

The TCGA and ArrayExpress datasets are publicly available, no ethical approval is required.

\section{Consent for publication}

Not Applicable.

\section{Competing interests}

None declared.

\section{Author details}

${ }^{1}$ Department of Urology, Affiliated Hospital of Nantong University, No.20 West Temple Road, Nantong, Jiangsu Province 226001, China. ${ }^{2}$ Department of Urology, The First Affiliated Hospital of Nanjing Medical University, Nanjing, Jiangsu Province 210029, China. ${ }^{3}$ Department of Urology, Zhongda Hospital Affiliated to Southeast University, Nanjing 210009, China.

Received: 9 August 2020 Accepted: 28 March 2021

Published online: 09 April 2021

\section{References}

1. Siegel RL, Miller KD, Jemal A. Cancer statistics, 2020. CA Cancer J Clin. 2020; 70(1):7-30. https://doi.org/10.3322/caac.21590.

2. Jonasch E, Gao J, Rathmell WK. Renal cell carcinoma. Bmj. 2014;349(nov10 11):g4797. https://doi.org/10.1136/bmj.g4797. 
3. Ljungberg B, Campbell SC, Cho HY, Jacqmin D, Lee JE, Weikert S, et al. The epidemiology of renal cell carcinoma. Eur Urol. 2011;60(4):615-21. https:// doi.org/10.1016/j.eururo.2011.06.049.

4. Warburg O. The metabolism of carcinoma cells. Cancer Res. 1925;9(1):14863. https://doi.org/10.1158/jcr.1925.148.

5. Koppenol WH, Bounds PL, Dang CV. Otto Warburg's contributions to current concepts of cancer metabolism. Nat Rev Cancer. 2011;11(5):325-37. https://doi.org/10.1038/nrc3038.

6. Akhtar M, Al-Bozom IA, Al Hussain T. Molecular and metabolic basis of clear cell carcinoma of the kidney. Adv Anat Pathol. 2018;25(3):189-96. https:// doi.org/10.1097/PAP.0000000000000185.

7. Nickerson ML, Jaeger E, Shi Y, Durocher JA, Mahurkar S, Zaridze D, et al. Improved identification of von Hippel-Lindau gene alterations in clear cell renal tumors. Clin Cancer Res. 2008;14(15):4726-34. https://doi.org/10.1158/1 078-0432.CCR-07-4921.

8. Srinivasan R, Ricketts CJ, Sourbier C, Linehan WM. New strategies in renal cell carcinoma: targeting the genetic and metabolic basis of disease. Clin Cancer Res. 2015;21(1):10-7. https://doi.org/10.1158/1078-0432.CCR-13-2993.

9. Yoshino H, Enokida H, Itesako T, Kojima S, Kinoshita T, Tatarano S, et al. Tumor-suppressive microRNA-143/145 cluster targets hexokinase-2 in renal cell carcinoma. Cancer Sci. 2013;104(12):1567-74. https://doi.org/10.1111/ca S. 12280 .

10. Li B, Qiu B, Lee DSM, Walton ZE, Ochocki JD, Mathew LK, et al. Fructose-1,6bisphosphatase opposes renal carcinoma progression. Nature. 2014; 513(7517):251-5. https://doi.org/10.1038/nature13557.

11. Song Y, Zheng S, Wang J, Long H, Fang L, Wang G, et al. Hypoxia-induced PLOD2 promotes proliferation, migration and invasion via PI3K/Akt signaling in glioma. Oncotarget. 2017;8(26):41947-62. https://doi.org/10.18632/oncota rget.16710.

12. Salem M, O'Brien JA, Bernaudo S, Shawer H, Ye G, Brkić J, et al. miR-590-3p promotes ovarian Cancer growth and metastasis via a novel FOXA2Versican pathway. Cancer Res. 2018;78(15):4175-90. https://doi.org/10.1158/ 0008-5472.CAN-17-3014.

13. Wu CT, Lin WY, Chen WC, Chen MF. Predictive value of CD44 in muscleinvasive bladder Cancer and its relationship with IL-6 signaling. Ann Surg Oncol. 2018;25(12):3518-26. https://doi.org/10.1245/s10434-018-6706-0.

14. Newman AM, Liu CL, Green MR, Gentles AJ, Feng W, Xu Y, et al. Robust enumeration of cell subsets from tissue expression profiles. Nat Methods. 2015;12(5):453-7. https://doi.org/10.1038/nmeth.3337.

15. Bray F, Ferlay J, Soerjomataram I, Siegel RL, Torre LA, Jemal A. Global cancer statistics 2018: GLOBOCAN estimates of incidence and mortality worldwide for 36 cancers in 185 countries. CA Cancer J Clin. 2018;68(6):394-424. https://doi.org/10.3322/caac.21492.

16. Chan DA, et al. Targeting GLUT1 and the Warburg effect in renal cell carcinoma by chemical synthetic lethality. Sci Transl Med. 2011;3(94):94ra70.

17. Jin $Y, H e J$, du J, Zhang RX, Yao HB, Shao QS. Overexpression of HS6ST2 is associated with poor prognosis in patients with gastric cancer. Oncol Lett. 2017;14(5):6191-7. https://doi.org/10.3892/ol.2017.6944.

18. Liep J, Kilic E, Meyer HA, Busch J, Jung K, Rabien A. Cooperative effect of miR-141-3p and miR-145-5p in the regulation of targets in clear cell renal cell carcinoma. PLoS One. 2016;11(6):e0157801. https://doi.org/10.1371/ journal.pone.0157801.

19. Shen T, et al. KIF20A affects the prognosis of bladder Cancer by promoting the proliferation and metastasis of bladder Cancer cells. Dis Markers. 2019; 2019:4863182

20. Sheng $Y$, Wang $W$, Hong $B$, Jiang $X$, Sun $R$, Yan $Q$, et al. Upregulation of KIF20A correlates with poor prognosis in gastric cancer. Cancer Manag Res. 2018;10:6205-16. https://doi.org/10.2147/CMAR.S176147.

21. Zhang Z, et al. Aberrant KIF20A expression is associated with adverse clinical outcome and promotes tumor progression in prostate Cancer. Dis Markers. 2019:2019:4782730.

22. Lu M, Huang X, Chen Y, Fu Y, Xu C, Xiang W, et al. Aberrant KIF20A expression might independently predict poor overall survival and recurrence-free survival of hepatocellular carcinoma. IUBMB Life. 2018;70(4): 328-35. https://doi.org/10.1002/iub.1726.

23. Xiong M, Zhuang K, Luo Y, Lai Q, Luo X, Fang Y, et al. KIF20A promotes cellular malignant behavior and enhances resistance to chemotherapy in colorectal cancer through regulation of the JAK/STAT3 signaling pathway. Aging (Albany NY). 2019;11(24):11905-21. https://doi.org/10.18632/aging.102 505.
24. Kawai Y, Shibata K, Sakata J, Suzuki S, Utsumi F, Niimi K, et al. KIF20A expression as a prognostic indicator and its possible involvement in the proliferation of ovarian clear-cell carcinoma cells. Oncol Rep. 2018;40(1):195205. https://doi.org/10.3892/or.2018.6401.

25. Zhao X, Zhou LL, Li X, Ni J, Chen P, Ma R, et al. Overexpression of KIF20A confers malignant phenotype of lung adenocarcinoma by promoting cell proliferation and inhibiting apoptosis. Cancer Med. 2018;7(9):4678-89. https://doi.org/10.1002/cam4.1710.

26. Tang J, Xu J, Zhi Z, Wang X, Wang Y, Zhou Y, et al. MiR-876-3p targets KIF20A to block JAK2/STAT3 pathway in glioma. Am J Transl Res. 2019;11(8): 4957-66.

27. Zhang W, He W, Shi Y, Gu H, Li M, Liu Z, et al. High expression of KIF2OA is associated with poor overall survival and tumor progression in early-stage cervical squamous cell carcinoma. PLoS One. 2016;11(12):e0167449. https:// doi.org/10.1371/journal.pone.0167449.

28. Mandal K, Pogoda K, Nandi S, Mathieu S, Kasri A, Klein E, et al. Role of a Kinesin Motor in Cancer Cell Mechanics. Nano Lett. 2019;19(11):7691-702. https://doi.org/10.1021/acs.nanolett.9b02592.

29. Asahara S, Takeda K, Yamao K, Maguchi H, Yamaue H. Phase I/II clinical trial using HLA-A24-restricted peptide vaccine derived from KIF20A for patients with advanced pancreatic cancer. J Transl Med. 2013;11(1):291. https://doi. org/10.1186/1479-5876-11-291.

30. Yang L, Wang L, Yang Z, Jin H, Zou Q, Zhan Q, et al. Up-regulation of EMTrelated gene VCAN by NPM1 mutant-driven TGF- $\beta / C P M L$ signalling promotes leukemia cell invasion. J Cancer. 2019;10(26):6570-83. https://doi. org/10.7150/jca.30223.

31. Kahlert UD, Joseph JV, Kruyt FAE. EMT- and MET-related processes in nonepithelial tumors: importance for disease progression, prognosis, and therapeutic opportunities. Mol Oncol. 2017;11(7):860-77. https://doi.org/10.1 002/1878-0261.12085.

32. Morais M, Dias F, Teixeira AL, Medeiros R. MicroRNAs and altered metabolism of clear cell renal cell carcinoma: potential role as aerobic glycolysis biomarkers. Biochim Biophys Acta Gen Subj. 2017;1861(9):217585. https://doi.org/10.1016/j.bbagen.2017.05.028.

33. Zhao W, Yang S, Chen J, Zhao J, Dong J. Forced overexpression of FBP1 inhibits proliferation and metastasis in cholangiocarcinoma cells via Wnt/ 3 catenin pathway. Life Sci. 2018;210:224-34. https://doi.org/10.1016/j.lfs.2018. 09.009.

34. Zhang YP, Liu KL, Yang Z, Lu BS, Qi JC, Han ZW, et al. The involvement of FBP1 in prostate cancer cell epithelial mesenchymal transition, invasion and metastasis by regulating the MAPK signaling pathway. Cell Cycle. 2019; 18(19):2432-46. https://doi.org/10.1080/15384101.2019.1648956.

35. Mitsui $Y$, Shiina H, Kato T, Maekawa S, Hashimoto Y, Shiina M, et al. Versican promotes tumor progression, metastasis and predicts poor prognosis in renal carcinoma. Mol Cancer Res. 2017;15(7):884-95. https://doi.org/10.11 58/1541-7786.MCR-16-0444.

36. Yeung TL, Leung CS, Wong KK, Samimi G, Thompson MS, Liu J, et al. TGF- $\beta$ modulates ovarian cancer invasion by upregulating CAF-derived versican in the tumor microenvironment. Cancer Res. 2013;73(16):5016-28. https://doi. org/10.1158/0008-5472.CAN-13-0023.

\section{Publisher's Note}

Springer Nature remains neutral with regard to jurisdictional claims in published maps and institutional affiliations.

Ready to submit your research? Choose BMC and benefit from:

- fast, convenient online submission

- thorough peer review by experienced researchers in your field

- rapid publication on acceptance

- support for research data, including large and complex data types

- gold Open Access which fosters wider collaboration and increased citations

- maximum visibility for your research: over $100 \mathrm{M}$ website views per year

At BMC, research is always in progress.

Learn more biomedcentral.com/submissions 